\title{
Aluminum Deposition at the Osteoid-Bone Interface An Epiphenomenon of the Osteomalacic State in Vitamin D-deficient Dogs
}

L. Darryl Quarles, Vincent W. Dennis, Hillel J. Gitelman, John M. Harrelson, and Marc K. Drezner Departments of Medicine, Physiology, Pathology, and Surgery, Duke University Medical Center, Durham, North Carolina 27710; and Department of Medicine, University of North Carolina, Chapel Hill, North Carolina 27514

\begin{abstract}
Although aluminum excess is an apparent pathogenetic factor underlying osteomalacia in dialysis-treated patients with chronic renal failure, the mechanism by which aluminum impairs bone mineralization is unclear. However, the observation that aluminum is present at osteoid-bone interfaces in bone biopsies of affected patients suggests that its presence at calcification fronts disturbs the cellular and/or physiochemical processes underlying normal mineralization. Alternatively, aluminum at osteoid-bone interfaces may reflect deposition in preexistent osteomalacic bone without direct effects on the mineralization process. We investigated whether aluminum accumulates preferentially in osteomalacic bone and, if so, whether deposition of aluminum occurs at calcification fronts and specifically inhibits mineralization.
\end{abstract}

Aluminum chloride $(1 \mathrm{mg} / \mathrm{kg})$ was administered intravenously three times per week for 3 wk to five normal and five vitamin D-deficient osteomalacic dogs. Before administration of aluminum the vitamin D-deficient dogs had biochemical and bone biopsy evidence of osteomalacia. Bone aluminum content in the osteomalacic dogs $(15.1 \pm 2.2 \mu \mathrm{g} / \mathrm{g})$ and the plasma aluminum concentration $(10.4 \pm 2.1 \mu \mathrm{g} / \mathrm{liter})$ were no different than those of normal dogs $(10.5 \pm 3.5 \mu \mathrm{g} / \mathrm{g}$ and $11.9 \pm 1.2$ $\mu \mathrm{g} /$ liter, respectively). After the 3 wk of aluminum administration the plasma phosphorus, parathyroid hormone, and 25hydroxyvitamin D concentrations were unchanged in normal and vitamin D-deficient dogs. Similarly, no alteration in bone histology occurred in either group. In contrast, bone aluminum content increased to a greater extent in the vitamin D-deficient $\operatorname{dogs}(390.3 \pm 24.3 \mu \mathrm{g} / \mathrm{g})$ than in the normal dogs $(73.6 \pm 10.6$ $\mu \mathrm{g} / \mathrm{g})$. Moreover, aluminum localized at the osteoid-bone interfaces of the osteomalacic bone in the vitamin D-deficient dogs, covering $42.9 \pm 9.2 \%$ of the osteoid-bone surface.

Further, in spite of continued aluminum chloride administration (1 $\mathrm{mg} / \mathrm{kg}$ two times per week), vitamin $D$ repletion of the vitamin D-deficient dogs for 11 wk resulted in normalization of their biochemistries. In addition, while normal dogs maintained normal bone histology during the period of continued aluminum administration, vitamin $D$ repletion of the vitamin D-deficient dogs induced healing of their bones. Indeed, the

Part of this work appeared in abstract form in 1984. Clin. Res. 32: 522.

Address reprint requests to Dr. Drezner, Duke University Medical Center, Durham, NC 27710.

Received for publication 18 June 1984 and in revised form 17 January 1985.

J. Clin. Invest.

(c) The American Society for Clinical Investigation, Inc.

0021-9738/85/05/1441/07 $\$ 1.00$

Volume 75, May 1985, 1441-1447 appearance of aluminum in the cement lines of the healed bones indicated that mineralization had occurred at sites of prior aluminum deposition.

These observations illustrate that aluminum deposition in osteomalacic bone may be a secondary event that does not influence bone mineralization. Thus, although aluminum may cause osteomalacia in chronic renal failure, its presence at mineralization fronts may not be the mechanism underlying this derangement.

\section{Introduction}

A variety of studies have implicated aluminum in the pathogenesis of the low turnover osteomalacia that occurs in a minority of patients with renal osteodystrophy. Epidemiological observations have shown that a high prevalence of this bone disease exists in patients undergoing hemodialysis with water of high aluminum content (1-5). In addition, the severity of the osteomalacia has been strongly correlated with the aluminum content of the bone $(6,7)$. Moreover, several investigators have directly evaluated the potential for aluminum-induced bone toxicity using animal model systems. In these studies aluminum loading has been associated with the development of defective mineralization of bone epiphyses (8) as well as with induction of osteomalacic changes in the trabecular bone of rats and dogs (9-11).

Although these observations indicate that aluminum may cause the low turnover osteomalacia of chronic renal failure, the mechanism by which aluminum impairs bone mineralization remains uncertain. However, the recent observation that aluminum is present at the nonmineralizing osteoid-bone interfaces (calcification fronts) in bone biopsies from affected patients $(5,12-14)$ suggests that aluminum localizes at the mineralization front and directly inhibits calcification of osteoid. Such inhibition may result from the specific effects of aluminum or from effects dependent upon metabolic abnormalities that are a part of the uremic state. Alternatively, the presence of aluminum at the osteoid-bone interface may reflect deposition in preexistent osteomalacic bone, and the aluminum may have no direct effect on the mineralization process. Such a possibility is consistent with the recent clinical observations that indicate that aluminum-induced osteomalacia may be due, in part, to a relative deficiency of parathyroid hormone $(\mathrm{PTH})^{1}(15,16)$.

We designed the present study to determine whether aluminum accumulates preferentially in osteomalacic bone and, if so, whether deposition of aluminum occurs at calcification fronts and specifically inhibits mineralization. In these investigations we used vitamin D-deficient osteomalacic beagle puppies as a model of osteomalacia and tested effects of aluminum on the vitamin D-dependent mineralization process.

1. Abbreviations used in this paper: $25(\mathrm{OH}) \mathrm{D}, 25$-hydroxyvitamin $\mathrm{D}$; PTH, parathyroid hormone. 


\section{Methóds}

Study protocol. 3-mo-old beagle puppies, weighing $\sim 3 \mathrm{~kg}$, were randomly divided into two groups. During phase 1 of the study, group 1 pups $(n=5)$ received a normal diet containing $0.7 \%$ calcium, $0.5 \%$ phosphorus, and $2,200 \mathrm{U} / \mathrm{kg}$ vitamin $D_{3}$. In contrast, group 2 pups $(n=5)$ received a matched diet that was deficient in calcium $(0.4 \%)$ and vitamin D. The group 2 animals were housed under gold fluorescent light devoid of significant ultraviolet irradiation. After $15 \mathrm{wk}$ of dietary control we performed bone biopsies on group 1 and group 2 dogs (aged $6.4 \mathrm{mo}$ ). In addition, we obtained plasma for measurement of calcium, phosphorus, creatinine, PTH, 25-hydroxyvitamin D (25[OH]D), and aluminum. These studies served as a baseline for normal (group 1) and vitamin D-deficient (group 2) pups.

In phase 2 of the study animals in both groups received aluminum chloride $(1 \mathrm{mg} / \mathrm{kg})$ intravenously three times per week for $3 \mathrm{wk}$. During this treatment period group 1 and group 2 dogs continued receiving normal and calcium/vitamin D-deficient diets, respectively. At the conclusion of this phase we repeated bone biopsies and plasma biochemistries.

During phase 3 of the study, aluminum chloride administration was continued but both group 1 and group 2 animals received the normal diet replete in calcium and vitamin $D$. All animals were housed in quarters with ultraviolet light. During the 11 wk of phase 3 we continued aluminum chloride administration at a reduced dose $(1 \mathrm{mg} /$ kg intravenously, twice a week) to adjust the plasma aluminum concentration (Table I) to levels more comparable to those observed in patients with osteomalacia and chronic renal failure (12). At the conclusion of phase 3 of the study, bone biopsies and plasma biochemistries were repeated in dogs, then aged $9.8 \mathrm{mo}$

Biochemical studies. Plasma calcium and phosphorus were measured by colorimetric techniques using an autoanalyzer (Technicon Instruments Corp., Tarrytown, NY). Plasma creatinine was determined by an enzymatic assay (17). We measured the plasma PTH concentration using a radioimmunoassay kit (Diagnostic Systems Laboratories, Inc., Webster, TX) designed to measure the carboxy-terminal portion of the molecule according to previously described methods (18). Duplicate sarnples obtained from pups during each phase of the study were measured simultaneously. The intraassay coefficient of variation for the measurements was $10 \%$. The plasma $25(\mathrm{OH}) \mathrm{D}$ concentration was measured in triplicate samples by a modification of the methods of Haddad and Chyu (19). The interassay coefficient of variation averaged 8.9\%. The ionized calcium level was determined using an ICAI analyzer (Radiometer Co., Copenhagen, Denmark), and values were corrected to a $\mathrm{pH}$ of 7.4 .
We assayed plasma aluminum concentration according to previously described methods (20) using a flameless atomic absorption spectrophotometer (Perkin-Elmer Corp., Instrument Div., Norwalk, CT) with a graphite furnace. Interference was minimized by using a diluent containing ammonium hydroxide. During phase 2 and 3 of the study, when aluminum chloride was administered, the average plasma aluminum level was estimated by measuring the plasma concentration at variable intervals for 48-72 $\mathrm{h}$ after an intravenous injection and determining the area under the plasma concentration versus time curve, using the trapezoidal rule, divided by the interval between doses (21).

Bone siudies. Transcortical bone biopsies were obtained from the anterior iliac crest of dogs under general anesthesia. Chlortetracycline ( $250 \mathrm{mg}$ orally, twice daily) was administered to each dog over a 3-d period from day 21 to day 18 before biopsy and demeclocycline hydrochloride ( $150 \mathrm{mg}$ orally, three times daily) from day 6 to day 3 before biopsy. Bone specimens were fixed in ethanol and embedded in methylmethacrylate, unstained or prestained by the methods of Villanueva (22). 20- and 5- $\mu \mathrm{m}$ sections were made and prepared for histomorphological examination according to previously published methods (23). Staining for aluminum was performed on $5-\mu \mathrm{m}$ prestained and unstained sections by a modification of methods reported by Maloney et al. (13)

Histomorphometric analysis of the trabecular bone in each section was accomplished by examining 100 fields with an integrated reticle (Merz-Schenk, Wild, Heerbrugg, Switzerland) (24). The following histological functions were quantitated: 1) mineralization front activity, the percentage of osteoid-covered trabecular bone surface exhibiting a fluorescent demeclocycline hydrochloride label with a minimum width of $3 \mu \mathrm{m}$;2) mineral apposition rate, the average distance between chlortetracycline and demeclocycline hydrochloride fluorescent labels divided by the days elapsed between the administration of the labels; 3) mean osteoid seam width, the mean width of 50 randomly selected osteoid seams, each measured at four points equidistant from each other using a linear reticle calibrated with a stage micrometer; 4) osteoid surface, the percentage of trabecular bone surface covered by osteoid; 5) active resorption surface, the percentage of nonosteoidcovered trabecular bone surface on which Howship's lacunae containing multinucleated osteoclasts are present; 6) osteoblastic surface, the percentage of trabecular bone surface on which osteoblast-covered osteoid is present; 7) relative osteoid volume, the percentage of trabecular bone volume composed of unmineralized bone; 8) bone volume, the percentage of the sample composed of trabecular bone; and 9) aluminum surface, the percentage of osteoid-covered trabecular bone surface bearing aluminum-stained osteoid-bone interfaces. In

Table I. Plasma Biochemistries of Group 1 and Group 2 Pups during Phases 1-3 of the Study

\begin{tabular}{clllllll}
\hline & Calcium & iCalcium & Phosphorus & PTH & $25(\mathrm{OH}) \mathrm{D}$ & $\begin{array}{l}\text { Alkaline } \\
\text { phosphatase }\end{array}$ & Aluminum \\
\hline & mmol/liter & mmol/liter & $m m o l / l i t e r$ & $n g / m l$ & $n g / m l$ & $I U / l i t e r$ & $\mu g / l i t e r$ \\
Phase 1 & & & & & & \\
Group 1 & $2.70 \pm 0.03$ & $1.41 \pm 0.01$ & $1.98 \pm 0.03$ & $0.49 \pm 0.03$ & $49.0 \pm 6.6$ & $56.6 \pm 2.8$ & $11.9 \pm 1.2$ \\
Group 2 & $1.60 \pm 0.15^{*}$ & $0.86 \pm 0.07^{*}$ & $1.57 \pm 0.06^{*}$ & $0.88 \pm 0.05^{*}$ & $15.6 \pm 1.3^{*}$ & $164.2 \pm 5.5^{*}$ & $10.4 \pm 2.1$ \\
Phase 2 & & & & & & & \\
Group 1 & $2.71 \pm 0.09$ & $1.39 \pm 0.02$ & $1.89 \pm 0.10$ & $0.50 \pm 0.02$ & $51.4 \pm 7.7$ & $70.3 \pm 11.5$ & $4,373.2 \pm 604.9^{*} \ddagger$ \\
Group 2 & $2.09 \pm 0.09^{*} \ddagger$ & $1.02 \pm 0.06^{*} \ddagger$ & $1.36 \pm 0.06^{*}$ & $0.86 \pm 0.07^{*}$ & $16.2 \pm 1.7^{*}$ & $137.6 \pm 10.8^{*}$ & $2,347.8 \pm 129.8^{*} \ddagger$ \\
Phase 3 & & & & & & & \\
Group 1 & $2.69 \pm 0.04$ & $1.37 \pm 0.01$ & $2.06 \pm 0.10$ & $0.41 \pm 0.02$ & $70.2 \pm 15.5$ & $46.2 \pm 7.4$ & $1,340.6 \pm 60.6 \S$ \\
Group 2 & $2.66 \pm 0.02 \S$ & $1.36 \pm 0.01 \S$ & $2.34 \pm 0.23 \S$ & $0.42 \pm 0.05 \S$ & $83.7 \pm 20.5 \S$ & $47.1 \pm 7.1 \S$ & $1,209.7 \pm 46.1 \S$
\end{tabular}

All values expressed as mean \pm SEM. * Significantly different from group 1 at $P<0.05$. $\ddagger$ Significantly different from value of comparable group in phase 1 at $P<0.05$. § Significantly different from values of comparable group during phase 1 and phase 2 at $P<0.05$. 
addition, we calculated the following according to previously published methods (25): 1) mineralization lag time, a measure of the time (in days) during the life span of an osteoid moiety when mineralization is not occurring; 2) corrected mineral apposition rate, the rate of mineral apposition averaged throughout the life span of the osteoid seams; 3) bone formation rate (formation surface referent), the volume of mineralized new bone formed per unit area of osteoid surface per unit time; and 4) bone formation rate (volume referent), the volume of mineralized new bone made per unit volume of preexisting bone per unit of time. Measurement of these variables in group 1 animals provided normal values during phase 1 of the study. After aluminum administration, however, we compared values obtained during phases 2 and 3 in group 1 pups with those determined in bone biopsies from three age-matched beagle puppies maintained on a normal diet and untreated with aluminum. Since no significant differences were observed at 7.75 or 9.8 mo of age, we limited comparison in phases 2 and 3 of the study to the data obtained from bone biopsies of group 1 (normal) and group 2 pups.

We measured bone aluminum content as follows. Samples were washed with a high pressure stream of aluminum-free water until completely devoid of marrow. Specimens were subsequently treated in a muffle furnace at $550^{\circ} \mathrm{C}$ for $12-16 \mathrm{~h}$, the dry weight of each sample was determined, and ashed specimens dissolved in $2 \mathrm{ml}$ of $3 \%$ ultrapure nitric acid. After bringing the samples to $10 \mathrm{ml}$ with deionized water, aliquots were assayed for aluminum content (20).

Statistical analyses. Results are expressed as the mean \pm SEM. We performed statistical analyses of the data obtained in group 1 and 2 dogs during the three phases of study by two-way analysis of variance and the Duncan's multiple range test (26).

Materials. The authentic $25(\mathrm{OH}) \mathrm{D}$ used in the assay for this metabolite was a gift from Dr. Milan Uskokovic, Hoffmann-La Roche Inc., Nutley, NJ. [ $\left.{ }^{3} \mathrm{H}\right] 25(\mathrm{OH}) \mathrm{D}_{3}(90 \mathrm{Ci} / \mathrm{mmol})$ was purchased from Amersham Corp. (Arlington Heights, IL). Beagle puppies were obtained from Ridglan Farms (Mount Horeb, WI) and normal and calcium/ vitamin $\mathrm{D}_{3}$-deficient diets from Teklad (Madison, WI).

\section{Results}

Phase 1. After $15 \mathrm{wk}$ of a calcium and vitamin D-deficient diet, group 2 pups displayed biochemical abnormalities characteristic of vitamin D deficiency (Table I). The plasma calcium and phosphorus concentrations of group 2 animals were significantly less than those of group 1 normal animals. In accord, group 2 dogs manifested an elevated plasma PTH level. Moreover, the plasma 25(OH)D concentration of the vitamin D-depleted pups was significantly less than that of the normal pups. In addition, an elevated plasma alkaline phosphatase concentration in group 2 dogs suggested that the vitamin $\mathrm{D}$ deprivation had resulted in development of osteomalacia.

Quantitative histomorphometric analysis of bone biopsies confirmed the presence of osteomalacia in the vitamin Ddeprived dogs (Table II). Representative sections demonstrated excessive osteoid surface and relative osteoid volume, changes characteristic of the osteomalacic state. In addition, mineralization dynamics were typically abnormal, resulting in a significantly decreased mineralization front activity and mineral apposition rate. Moreover, group 2 pups displayed a prolonged mineralization lag time compared with group 1 animals. Consistent with an elevated plasma PTH concentration, group 2 pups also manifested a 2.5 -fold increase of active bone resorption. However, the mineralized bone formation rate was significantly reduced (Table II). In contrast, osteoblastic surface of the group 2 pups was no different from that of group 1 pups (Table II).
In spite of these differences in bone histology, the bone aluminum content was not significantly different between groups 1 and 2 (Table II), and plasma aluminum levels were indistinguishable (Table I). Histochemical staining of the bone for aluminum revealed no evident deposition.

Phase 2. Parenteral administration of aluminum for $3 \mathrm{wk}$ resulted in no significant change in any of the measured biochemistries in the group 1 animals (Table I). In contrast, group 2 dogs showed a significant increase in both the plasma ionized and total calcium concentration, while the plasma phosphorus, PTH, 25(OH)D levels, and alkaline phosphatase activity did not significantly deviate from values in phase 1 (Table I).

Bone histology in both groups after aluminum exposure revealed changes consistent only with aging (Table II). Corrected mineral apposition rate in group 1 animals decreased from $1.60 \pm 0.1$ to $1.25 \pm 0.04 \mu \mathrm{m} / \mathrm{d}$ and bone formation rate (volume referent) from $1.18 \pm 0.1$ to $0.84 \pm 0.11 \mathrm{~mm}^{3} / \mathrm{mm}^{3}$ per yr. While these changes represent significant declines, untreated pups of the same age $(7.25 \mathrm{mo})$ had a mineral apposition rate and bone formation rate that are not significantly different, $1.42 \pm 0.18 \mu \mathrm{m} / \mathrm{d}$ and $0.67 \pm 0.10 \mathrm{~mm}^{3} / \mathrm{mm}^{3}$ per yr (Table II). In contrast, mineralization front activity, osteoid surface, mean osteoid seam width, and relative osteoid volume were unchanged.

In any case, administration of aluminum significantly increased the plasma aluminum concentration and bone aluminum content in both group 1 and 2 dogs (Table II). However, the bone aluminum content of group 2 osteomalacic pups $(390.3 \pm 24.3 \mu \mathrm{g} / \mathrm{g})$ increased to a level greater than five times that observed in the bones of group 1 normal pups $(73.6 \pm 10.6 \mu \mathrm{g} / \mathrm{g})$. In accord, the plasma aluminum concentration in the osteomalacic dogs was less than that in normal dogs (Table I). Commensurately, histological staining of the bone biopsies from group 2 pups revealed bright red aluminum bands covering $42.9 \pm 9.2 \%$ of the osteoid-bone interface (Table I; Fig. 1), but there was no evidence of aluminum staining in the bones of group 1 animals.

Phase 3. Continued administration of aluminum for an additional $11 \mathrm{wk}$, albeit at a reduced dosage, did not alter the plasma biochemistries or bone histology in the group 1 dogs that remained on a normal diet (Tables I and II). In contrast, provision of a calcium/vitamin $\mathrm{D}_{3}$-replete diet to the dogs in group 2 and reduction of aluminum dosage resulted in normal plasma biochemistries and healing of the osteomalacia. Plasma calcium and phosphorus concentrations increased significantly from the values observed in phase 1 and 2 to levels indistinguishable from those of group 1 dogs. Further, the plasma PTH in group 2 dogs decreased to normal, and the plasma $25(\mathrm{OH}) \mathrm{D}$ concentration returned to a normal level that was not significantly different from that of group 1 dogs. In addition, the plasma alkaline phosphatase activity of group 2 dogs decreased to the normal range.

In accord, maintenance of group 2 animals on a calcium/ vitamin $\mathrm{D}_{3}$-replete diet resulted in changes in both static and dynamic bone histomorphometric parameters indicative of bone healing. Despite continued administration of aluminum, albeit at a lower dosage, bone mineralization was normalized in group 2 animals as evidenced by labeling frequencies (mineralization front activity, $87.5 \pm 2.7 \%$, Table II) and interlabel distance (mineral apposition rate, $1.41 \pm 0.12 \mu \mathrm{m} / \mathrm{d}$ ). In accord, mineralized bone formation rates increased to levels 


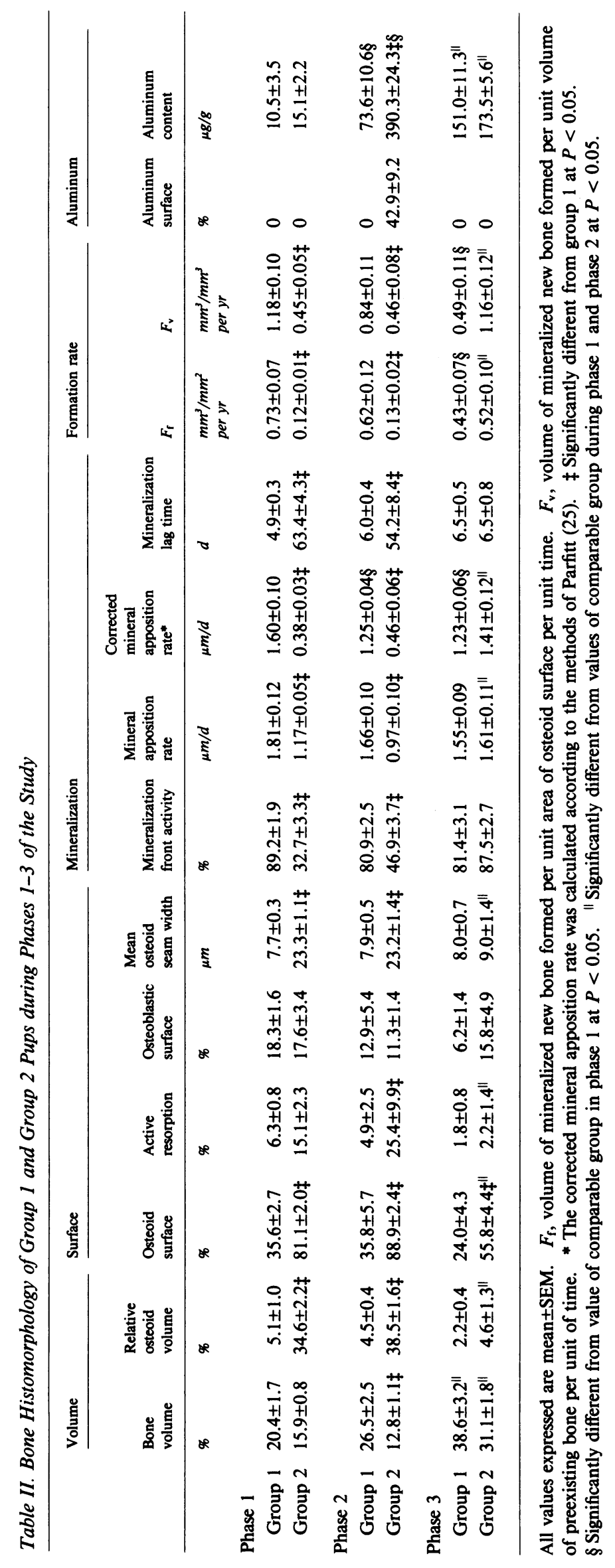

1444 Quarles, Dennis, Gitelman, Harrelson, and Drezner 

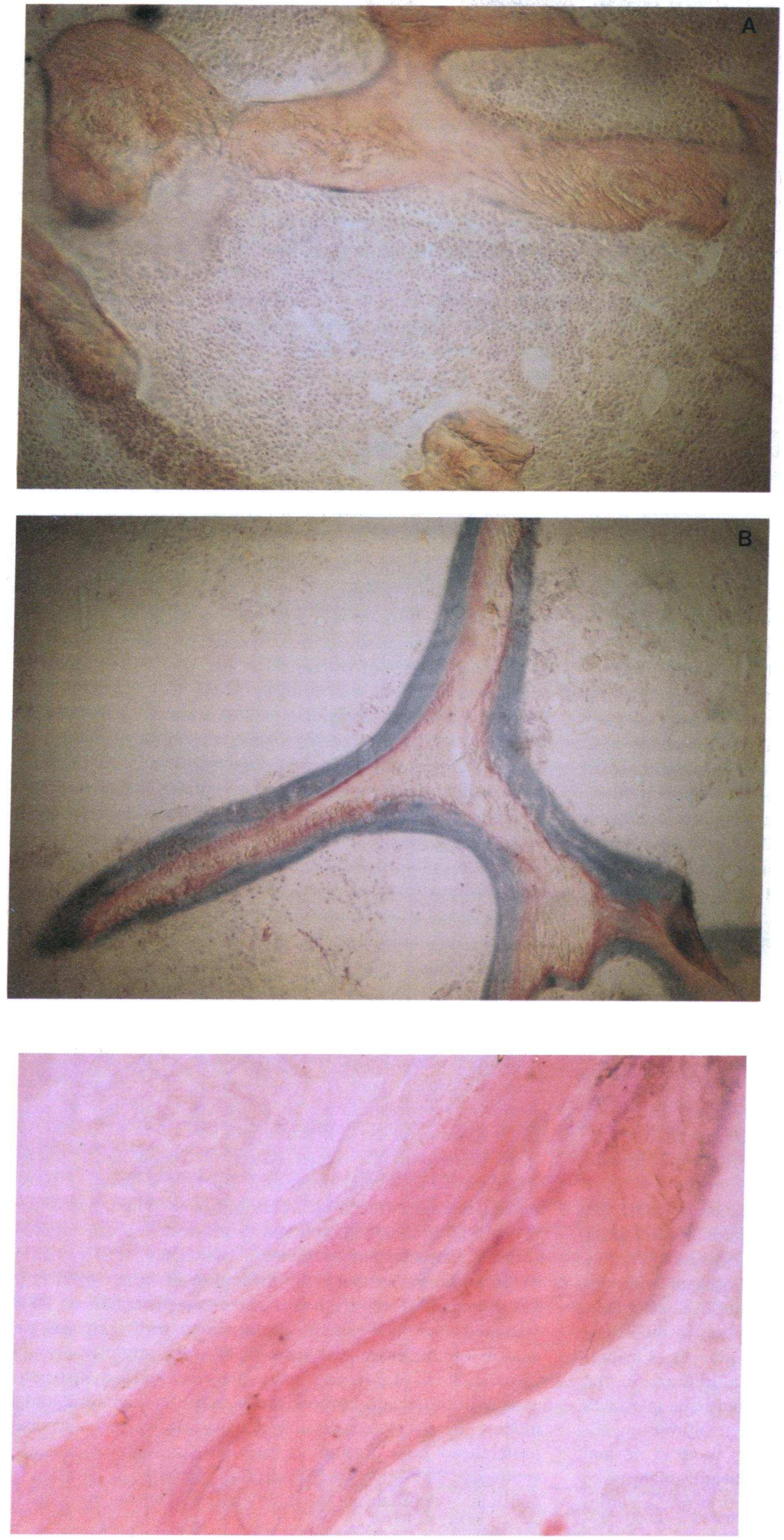

Figure 1. (top) Microscopic appearance of bone biopsies from normal (group 1) and vitamin D-deficient osteomalacic (group 2) dogs after aluminum administration. $(A)$ Villanueva-stained specimen from a normal dog revealing little blue-stained osteoid. Aluminum stain did not disclose any demonstrable aluminum at osteoid-bone interfaces. $(B)$ Villanueva-stained specimen from a vitamin D-deficient (group 2) dog after aluminum administration. Bluestained osteoid is abundant and covers the majority of the trabecular bone surface. In addition, red-stained aluminum is evident over a large portion of the osteoid-bone interface.

Figure 2. (bottom) Microscopic appearance of a bone biopsy from a group 2 dog after vitamin $D$ repletion and continued aluminum administration. A previously un-

stained 5- $\mu \mathrm{m}$ section, reacted with a histochemical stain, has evident red-stained aluminum in a cement line. The presence of aluminum in the cement line indicates that mineralization had occurred at an osteoidbone interface where aluminum had been previously deposited. 
significantly greater than those in group 1 dogs. The appearance of the fluorescent bands in a discrete double linear pattern further confirmed normal mineralization dynamics. In addition, the mean osteoid seam width had decreased significantly into the normal range with the consequence that mineralization lag time had normalized. Concomitant improvements of osteoid surface and relative osteoid volume were also observed, although the mean values remained above those of group 1 dogs (Table II).

The additional $11 \mathrm{wk}$ of aluminum exposure further increased the bone aluminum content of the group 1 dogs (73.6 \pm 10.6 vs. $151.0 \pm 11.3 \mu \mathrm{g} / \mathrm{g}$; Table II). In contrast, healing of the osteomalacia in group 2 animals was associated with a significant decrease of the bone aluminum content $(390.3 \pm 24.3$ vs. $173.5 \pm 5.6 \mu \mathrm{g} / \mathrm{g}$; Table II) to a level indistinguishable from that in group 1 . Histochemical staining again revealed no apparent aluminum in group 1 animals. Bone biopsies from group 2 dogs that previously had identifiable aluminum at the mineralization fronts then displayed red stained metal only in cement lines (Fig. 2), indicating that mineralization occurred over the previous sites of aluminum deposition.

\section{Discussion}

Considerable evidence indicates that aluminum is a pathogenetic factor underlying the low turnover osteomalacia in dialysis-treated patients with chronic renal failure. The mechanism for the disordered bone mineralization induced by aluminum excess, however, remains unclear. Several observations suggest that the local effects of aluminum on bone mineralization dynamics are essential to the development of the osteomalacia. Primary among these is the demonstration that aluminum is concentrated at the osteoid-bone interface (calcification front) in affected bones $(5,12-14)$. A consequent disturbance of cellular and/or physiochemical processes at this site may result in the abnormal mineralization. The association of decreased bone aluminum content with the beneficial effects of desferreoxamine on bone healing in affected subjects supports this possibility (27).

However, the results of this investigation illustrate that aluminum accumulates preferentially in preexistent osteomalacic bone, localizes at the osteoid-bone interfaces, and does not prevent mineralization. In contrast, aluminum accumulation in exposed normal bones is significantly less and is not histologically evident at the osteoid-bone interfaces. Thus, the mere presence of aluminum at the calcification front (osteoidbone interface) in the osteomalacic bone does not impair vitamin D-dependent mineralization.

In response to parenterally administered aluminum, the bones of vitamin D-deficient osteomalacic pups accumulated aluminum to an extent more than five times greater than that in the bones of normal dogs (Table II). Consistent with the enhanced accumulation, the plasma aluminum level, estimated from pharmacokinetic data, was significantly less in the osteomalacic pups despite similar exposure (Table I). Further, histological examination revealed that the aluminum in the osteomalacic bone covered $\sim 43 \%$ of the osteoid-bone interfaces where initial mineralization occurs. However, aluminum administration did not affect mineralization of bone in normal or D-deficient pups (Table II). No significant changes in mineralization front activity, mineral apposition rate, or min- eralization lag time occurred in group 1 or 2 pups after administration of aluminum for 3 wk.

More important, the presence of aluminum at the osteoidbone interface in the bones of osteomalacic pups did not interfere with remineralization of bones upon vitamin $D$ repletion. In spite of continued aluminum administration at a reduced dosage, vitamin $\mathrm{D}$ therapy resulted in normalization of mineralization and improved static parameters of bone histology. Mineralization front activity and mineralization lag time returned to the normal range. Indeed, the presence of aluminum in bone cement lines (Fig. 2) indicated that the mineralization had occurred at sites of aluminum deposition. The similar finding of aluminum in the cement lines of bone from humans with renal osteodystrophy is consistent with our observations (13). In association with normal mineralization dynamics, mean osteoid seam width was normal, while relative osteoid volume and osteoid surface were markedly reduced (Table II). The persistence of slightly abnormal relative osteoid volume and osteoid surface probably represents the short duration of treatment.

In this study, we did not observe aluminum-induced osteomalacia in the normal dogs, results that are similar to previous investigations (10). In contrast, several other studies have reported that aluminum administration to rats and dogs results in the development of bone disease $(8,9,11)$. In general these disparate observations may be related to several variables. First, the induction of osteomalacia has been associated with using either a total dose of aluminum significantly greater than that which we used $(8,9)$ or a greater amount over a shorter period (11). We observed a progressive accumulation of aluminum in the bones of normal dogs with continuous administration in spite of reduced dosage in phase 3 . However, the plasma aluminum concentration fell by $>50 \%$ during this part of the study, potentially reducing the effects on osteoblasts. Accordingly, abnormal bone mineralization may have resulted from continued, or increased, aluminum administration. Second, in most of the studies in which aluminum treatment has induced osteomalacia, the experimental animals had impaired renal function (9-11). Since the major (or only) route of elimination for parenterally administered aluminum is the kidneys, a decrease in renal function no doubt amplifies the tissue burden for a given dose. All dogs in our study, however, maintained normal renal function throughout the investigation. Thus, the present studies do not preclude the possibility that aluminum administration may cause osteomalacia if given in higher doses or under different conditions.

In this regard the model system of osteomalacia that we investigated has several important differences from the osteomalacic disease induced by aluminum in chronic renal failure. Vitamin $\mathrm{D}$ deficiency in young dogs produced an osteomalacic disorder characterized by increased osteoclast activity (Table II). In addition, although the formation of mineralized bone was decreased (Table II), it is possible that the enhanced PTHmediated resorptive activity is associated with increased formation of unmineralized osteoid. We did not measure this variable, however. In any case, the osteomalacia associated with aluminum and chronic renal failure is adynamic and is marked by decreased cellular activity, as well as decreased mineralized bone formation rate and, undoubtedly, osteoid synthesis. The increased resorption activity of vitamin D deficiency osteomalacia, the maintenance of normal osteoblastic 
surface, and the probable enhanced rate of osteoid synthesis may facilitate aluminum deposition and/or counteract any effects the aluminum may have on bone mineralization. Moreover, we studied the effects of aluminum in the absence of the metabolic complications generally associated with reduced renal function. Such metabolic abnormalities may modulate aluminum effects on bone mineralization.

Nevertheless, our data illustrate that aluminum deposition in vitamin D-deficient osteomalacic bone may be a secondary event that does not influence bone mineralization. Therefore, although aluminum may cause osteomalacia in patients with chronic renal failure, the presence of aluminum deposits at the osteoid-bone interface of bones from affected patients with a characteristic low turnover osteomalacia is insufficient to establish the mechanism underlying the abnormal mineralization of aluminum-induced osteomalacia. Indeed, further studies may reveal that aluminum accumulation in body fluids and/ or organs (other than bone) causes osteomalacia by a mechanism distinctly different from the postulated direct effects of aluminum deposition on bone mineralization. In any case, additional data are necessary to establish the mechanism by which aluminum induces osteomalacia.

\section{Acknowledgments}

We are indebted to Ms. Janice Almond, Mr. Robert White, and Ms. Jeanne Raye for excellent technical assistance, and Ms. Beth Gunn for typing the manuscript.

This study was supported in part by grants from the National Institute of Arthritis, Metabolism and Digestive Diseases (2 ROI AM27032, 5 PO1 CA11265, RO1 AM26201) and by a research grant from the National Foundation (1-852). Dr. Drezner is a recipient of a Research Career Development Award (5 KO4 AM00643) from the National Institute of Arthritis, Metabolism and Digestive Diseases.

\section{References}

1. Platts, M. M., G. C. Hoods, and J. S. Hislop. 1977. Composition of the domestic water supply and the incidence of fractures and encephalopathy in patients on home dialysis. Br. Med. J. 2:657-660.

2. Ward, M. K., T. G. Feest, H. A. Ellis, I. S. Parkinson, and D. N. S. Kerr. 1978. Osteomalacic dialysis osteodystrophy: evidence for a water-borne etiologic agent, probably aluminum. Lancet. I:841845.

3. Pierides, A. M., W. G. Edwards, Jr., U. S. Cullu, Jr., J. T. McCall, and H. A. Ellis. 1980. Hemodialysis encephalopathy with osteomalacic fractures and muscle weakness. Kidney Int. 18:115-124.

4. Walker, G. S., J. E. Aaron, M. Peacock, P. J. A. Robinson, and A. M. Davison. 1982. Dialysate aluminum concentration and renal bone disease. Kidney Int. 21:411-415.

5. McClue, J., N. L. Fazzalarf, R. G. Fassett, and D. J. Pugsley. 1983. Bone histoquantitative findings and histochemical staining reactions for aluminum in chronic renal failure patients treated with haemodialysis fluids containing high and low concentrations of aluminum. J. Clin. Pathol. (Lond.). 36:1281-1287.

6. Hodsman, A. B., D. J. Sherrard, A. C. Alfrey, S. M. Ott, A. S. Brickman, M. L. Miller, N. A. Maloney, and J. W. Coburn. 1982. Bone aluminum and histomorphometric features of renal osteodystrophy. J. Clin. Endocrinol. Metab. 54:539-546.

7. Ott, S. M., N. A. Maloney, J. W. Coburn, A. C. Alfrey, and D. J. Sherrard. 1982. The prevalence of aluminum in renal osteodystrophy and its relationship to response to calcitriol therapy. $N$. Engl. J. Med. 307:709-713.

8. Ellis, H. A., J. H. McCarthy, and J. Herrington. 1979. Bone aluminum in hemodialized patients and in rats injected with aluminum chloride: relationship to impaired bone mineralization. J. Clin. Pathol. (Lond.). 32:832-844.

9. Robertson, J. A., A. J. Felsenfeld, C. C. Haygood, P. Wilson, C. Clark, and F. Llach. 1983. An animal model of aluminum-induced osteomalacia: role of chronic renal failure. Kidney Int. 23:327-335.

10. Chan, Y., A. C. Alfrey, S. Posen, D. Lissner, E. Hills, C. R. Dustan, and R. A. Evans. 1983. The effect of aluminum on normal and uremic rats: tissue distribution, vitamin $\mathrm{D}$ metabolites and quantitative bone histology. Calcif. Tissue Int. 23:344-351.

11. Goodman, W. G., D. A. Henry, R. Horst, R. K. Nudelman, A. C. Alfrey, and J. W. Coburn. 1984. Parenteral aluminum administration in the dog: II. Induction of osteomalacia and effect on vitamin D metabolism. Kidney Int. 25:370-375.

12. Cournot-Witmer, G., J. Zingraff, J. J. Plachot, F. Escaig, R. Lefevre, P. Boumati, A. Bourdeau, M. Garabedian, P. Galle, R. Bourbon, T. Drueke, and S. Balsan. 1981. Aluminum localization in bone from hemodialized patients: relationship to matrix mineralization. Kidney Int. 20:376-385.

13. Maloney, N. A., S. M. Ott, A. C. Alfrey, N. L. Miller, J. W. Coburn, and D. J. Sherrard. 1982. Histological quantitation of aluminum in iliac bone from patients with renal failure. J. Lab. Clin. Med. 99: 206-216.

14. Smith, P. S., and J. McClure. 1982. Localization of aluminum by histochemical and electron probe $\mathrm{x}$-ray microanalytical techniques in bone tissue of cases of renal osteodystrophy. J. Clin. Pathol. (Lond.). 35:1283-1293.

15. Hodsman, A. B., D. J. Sherrard, E. G. C. Wong, A. D. Brickman, D. B. N. Lee, A. C. Alfrey, F. R. Singer, A. W. Norman, and J. W. Coburn. 1981. Vitamin D-resistant osteomalacia in hemodialysis patients lacking secondary hyperparathyroidism. Ann. Intern. Med. 94:629-637.

16. Felsenfeld, A. J., J. M. Harrelson, R. A. Gutman, S. A. Wells, Jr., and M. K. Drezner. 1982. Osteomalacia after parathyroidectomy in patients with uremia. Ann. Intern. Med. 96:34-39.

17. Toffaletti, J., N. Blosser, T. Hall, S. Smith, and D. Tompkins. 1983. An automated dry-slide enzymatic method evaluated for measurement of creatinine in serum. Clin. Chem. 29:684-687.

18. Potts, J. T., L. J. Deftos, R. M. Buckle, L. M. Sherwood, and G. D. Aurbach. 1968. RIA of PTH: studies of the control of secretion of the hormone and parathyroid function in clinical disorders. In Radioisotopes in Medicine. U. S. Atomic Energy Commission, Washington DC. 207-229.

19. Haddad, J. G., Jr., and K. J. Chyu. 1971. Competitive proteinbinding radioassay for 25-hydroxycholecalciferol. J. Clin. Endocrinol. Metab. 33:992-995.

20. Alderman, F. R., and H. J. Gitelman. 1980. Improved electrothermal determination of aluminum in serum by atomic absorption spectroscopy. Clin. Chem. 26:258-260.

21. Gibaldi, M., and D. Perrier. 1975. Trapezoidal rule. In Pharmcokinetics. Marcel Dekker Inc., New York. 293-296.

22. Villanueva, A. R. 1974. A bone stain for osteoid seams in fresh, unembedded, mineralized bone. Stain Technol. 49:1-8.

23. Lyles, K. W., J. M. Harrelson, and M. K. Drezner. 1982. The efficacy of vitamin $D_{2}$ and oral phosphorus therapy in X-linked hypophosphatemic rickets and osteomalacia. J. Clin. Endocrinol. Metab. 54:307-315.

24. Merz, W. A., and R. K. Schenk. 1970. Quantitative structural analysis of human cancellous bone. Acta Anat. 75:54-66.

25. Parfitt, A. M. 1983. The physiologic and clinical significance of bone histomorphometric data. In Bone Histomorphometry: Techniques and Interpretation. CRC Press Inc., Boca Raton, FL. 143-223.

26. Neter, J., and W. Wasserman. 1974. Single factor analysis of variance. In Applied Linear Statistical Models. Richard D. Irwin Inc., Homewood, IL. 419-492.

27. Brown, D. J., J. K. Dawborn, K. N. Ham, and J. M. Xipell. 1982. Treatment of dialysis osteomalacia with desferroxamine. Lancet. II:343-345. 